\title{
Hypoplastic Left Heart Syndrome: An Image of Extreme
}

\section{Case}

\author{
F Bizzarri, MD*, PM de Siena, MD, S Congiu, MD, C Van Doorn, MD and $O$ Jaber, MD \\ Consultant in Cardiothoracic Surgery, Sapienza University of Rome, Italy
}

It is a picture of a baby patient affected by the syndrome, showing the gross anatomy of the heart self with a big discrepancy between the dimension of the main pulmonary artery and the ascending aorta. In this case the circulation is totally dependent by the Botallus duct.

A baby 2 d.o. $2.97 \mathrm{Kg}$ has been admitted with an antenatal diagnosis of Hypoplastic Left Heart Syndrome (HLHS) to our Centre Figure 1. A gestational echo showed a severe aortic and mitral atresia picture Figure 2.

On the echo repeated at birth, a large pulmonary artery was depicted with a very small ascending aorta. The PDA was large filling the descending aorta and the aortic arch, tricuspid valve was mildly insufficient, right ventricular function good without RVOTO. Normal coronary pattern and no other anomalous findings were detected.

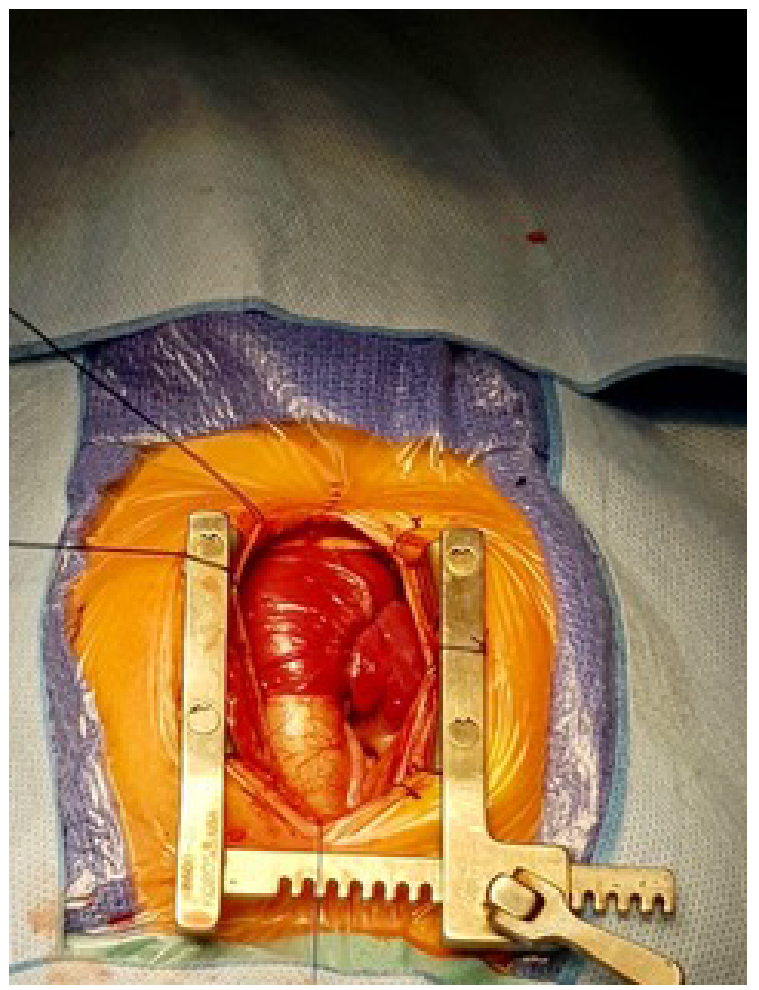

Figure 1: Antenatal diagnosis of Hypoplastic Left Heart Syndrome (HLHS).
At 36 w.o. birth he was haemodynamically stable, and was maintaining a relatively good systemic-pulmonary balance with $83 \%$ saturation. After $8 \mathrm{hrs}$. of age an infusion of alpoprostadil was started on, following severe desaturation and apnea requiring bagging, intubation, mechanical ventilation and paralysis in presence of PDA narrowing.

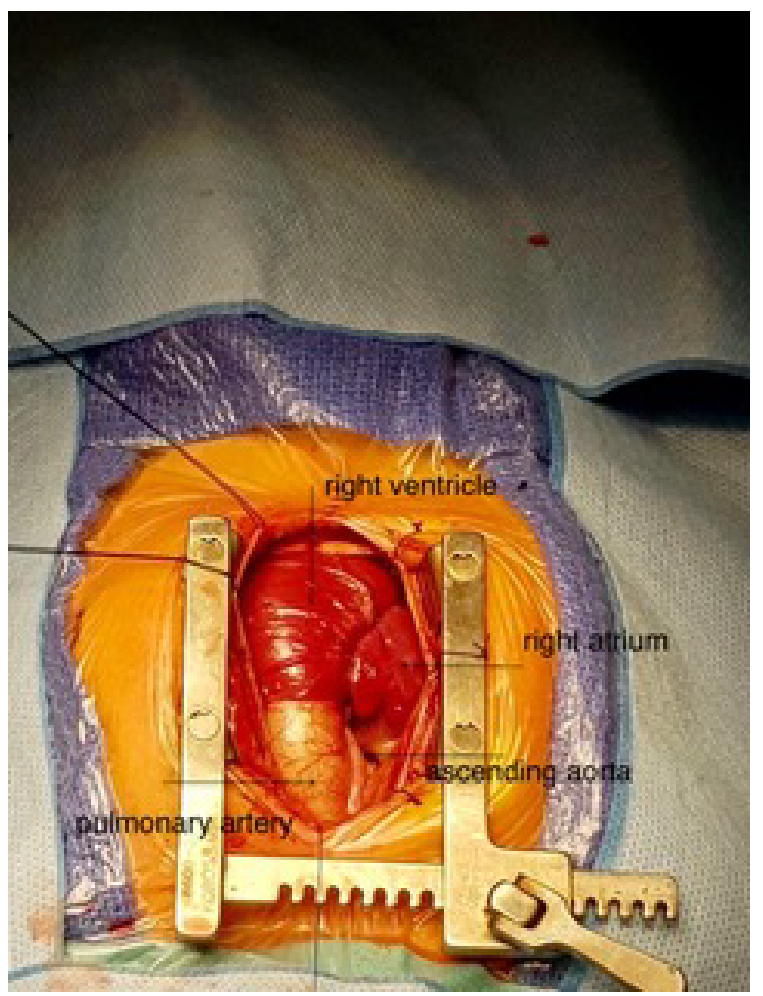

Figure 2: A gestational echo showing a severe aortic and mitral atresia. *Corresponding author: Federico Bizzarri, MD, Consultant in
Cardiothoracic Surgery, Sapienza University of Rome, Italy

Accepted: August 01, 2019

Published online: August 03, 2019

Citation: Bizzarri F, de Siena PM, S Congiu S, et al. (2019) Hypoplastic Left Heart Syndrome: An Image of Extreme Case. J Cardiothorac Surg Ther 3(1):35-36

Copyright: (C) 2019 Bizzarri F, et al. This is an open-access article distributed under the terms of the Creative Commons Attribution License, which permits unrestricted use, distribution, and reproduction in any medium, provided the original author and source are credited. 
On the $4^{\text {th }}$ day after birth he underwent a Norwood repair by using the Sano shunt technique with a $5 \mathrm{~mm}$. RV to PA shunt. The classical "Sano" technique was employed, and the chest was left open for $36 \mathrm{hrs}$. post-procedure with a small ligaclip left on the Sano shunt in order to reduce the pulmonary overflow following the pulmonary vascular resistance fall. The ligaclip was then removed at the chest closure.

The post-operative course was uneventful, and the patient was discharged on the $8^{\text {th }}$ post-operative day in good condition and stable haemodynamical status.

We present the operative pictures taken in operative room once the sternum and the pericardium open and the thymus glands totally resected.
We think that the pictures are useful to students and surgeons in training as educational images in order to take into account the surgical scenario faced in the operative room.

The pictures show an unexpected huge pulmonary artery compared to a filiform ascending aorta. The good pulmonary/ systemic haemodynamical balance, hold at birth, can be explained by the PDA filling an enlarged pulmonary artery until the PDA narrowing.

\section{Conflict of Interest}

I declare that I wrote the manuscript and I also disclosed any commercial association that might pose a conflict of interest in connection with the submitted article.

DOI: $10.36959 / 582 / 418$ 